Journal for ImmunoTherapy of Cancer

\section{metastatic prostate cancer with microsatellite instability high (MSI-H) detected by circulating tumor DNA}

To cite: Barata $\mathrm{P}$, Agarwal $\mathrm{N}$, Nussenzveig R, et al. Clinical activity of pembrolizumab in metastatic prostate cancer with microsatellite instability high (MSI-H) detected by circulating tumor DNA. Journal for ImmunoTherapy of Cancer 2020;8:e001065. doi:10.1136/ jitc-2020-001065

Accepted 19 July 2020
Check for updates

(c) Author(s) (or their employer(s)) 2020. Re-use permitted under CC BY-NC. No commercial re-use. See rights and permissions. Published by BMJ.

For numbered affiliations see end of article.

Correspondence to Dr Pedro Barata; pedrobaratamd@gmail.com

\section{ABSTRACT}

To report a multi-institutional case series of patients with advanced microsatellite instability high (MSI-H) prostate adenocarcinoma identified with clinical cell-free DNA (cfDNA) next-generation sequencing (NGS) testing and treated with immune checkpoint inhibitors. Retrospective analysis of patients with metastatic castration-resistant prostate cancer (mCRPC) and MSI-H tumor detected by a commercially available cfDNA NGS assay Guardant360 (G360, Guardant Health) at eight different Academic Institutions in the USA, from September 2018 to April 2020. From a total of $14 \mathrm{MSI}-\mathrm{H}$ metastatic prostate cancer patients at participating centers, nine patients with $\mathrm{mCRPC}$ with $56 \%$ bone, $33 \%$ nodal, $11 \%$ liver and $11 \%$ soft-tissue metastases and a median PSA of $29.3 \mathrm{ng} / \mathrm{dL}$, were treated with pembrolizumab after 2 lines of therapy for CRPC. The estimated median time on pembrolizumab was 9.9 (95\% Cl 1.0 to 18.8 ) months. Four patients (44\%) achieved PSA50 after a median of 4 (3-12) weeks after treatment initiation including three patients with $>99 \%$ PSA decline. Among the patients evaluable for radiographic response $(n=5)$, the response rate was $60 \%$ with one complete response and two partial responses. Best response was observed after a median of 3.3 (1.4-7.6) months. At time of cut-off, four patients were still on pembrolizumab while four patients discontinued therapy due to progressive disease and one due to COVID-19 infection. Half of the patients with PSA50 had both MSI-H and pathogenic alterations in BRCA1 and BRCA2 in their G360 assays. The use of liquid biopsy to identify metastatic prostate cancer patients with MSI-H is feasible in clinical practice and may overcome some of the obstacles associated with prostate cancer tumor tissue testing. The robust activity of pembrolizumab in selected patients supports the generalized testing for MSI-H.

\section{INTRODUCTION}

Immune-checkpoint blockade has shown promising therapeutic outcomes and several monoclonal antibodies that target cytotoxic T-lymphocyte-associated protein 4, programmed death 1 receptor (PD-1) and programmed death ligand 1 (PD-L1) have been approved for certain cancer treatments while others are under clinical investigation. ${ }^{12}$ Still, the activity of immunotherapy for unselected advanced prostate cancer, is in general, limited based on prospective phase II study. ${ }^{34}$

Mismatch repair (MMR) is a mechanism by which postreplicative mismatches in daughter DNA strands are repaired and replaced with the correct DNA sequence. Microsatellites, also known as short tandem repeats, consist of repeated sequences of 1-6 nucleotides. ${ }^{5}$ The mechanism of microsatellite generation is generally believed to be DNA slippage in the process of replication, or mismatch of the basic group of slippage strand and complementary strand in the process of DNA replication and repair, resulting in one or more of the repeating units missing or insert. ${ }^{5}$ MLH1, MSH2, MSH6 and PMS2 are all MMR enzymes that, when deficient, are associated with microsatellite instability (MSI) in cancer. $^{6}$

Historically, methods for MSI testing use tissue biopsies and include PCR-based amplification followed by capillary electrophoresis, and more recently next generation sequencing (NGS)-based approaches. ${ }^{7-9}$ Different assays using tumor tissue or plasma cell-free DNA (cfDNA) (liquid biopsies) are able to assess the MSI status in patients with cancers. ${ }^{1011}$ In prostate cancer, the prevalence of MSI-high (H)/deficient MMR (dMMR) has been observed in approximately $2 \%-3 \%$ of cases, based on different studies that used tumor tissue. ${ }^{11-13}$

MSI-H/dMMR tumors are often associated with greater and more durable responses after administration of immune checkpoint 
inhibitors. The humanized, anti-PD-1 monoclonal antibody pembrolizumab was the first tumor-agnostic approval in oncology by the US Food and Drug Administration, for tumors expressing dMMR or MSI-H, regardless of primary tumor and regardless of the underlying assay. ${ }^{12} 14-16$

We report a multi-institutional case series of advanced prostate cancer with MSI-H status identified with a commercially available cfDNA NGS assay and treated with immune checkpoint inhibitors. No prior experience has been reported using MSI-H circulating tumor DNA (ctDNA) as a predictive biomarker in prostate cancer for PD-1 inhibition.

\section{METHODS}

\section{Patients and samples}

A total of 14 patients with advanced prostate cancer treated at eight different Academic Institutions in the USA, had MSI-H status detected by a commercially available cfDNA NGS assay, Guardant360 (G360). All patients had G360 testing for advanced disease. Medical record review was conducted for patient clinical characteristics and outcomes, with follow-up through April 202020.

\section{Sequencing and analysis}

G360 (Guardant Health, Redwood City, California, USA) is a 74-gene panel validated for detection of single nucleotide variants (SNVs), indels, copy number amplifications, fusions and MSI-H status in cfDNA from plasma of patients with advanced solid tumors. ${ }^{11}{ }^{17}$ This panel started reporting MSI-H status on September 272018. G360 sequences 90 pan-cancer informative microsatellite loci from plasma cfDNA and reports MSI-H status based on the number of detected unstable sites exceeding a predetermined threshold, as previously described. ${ }^{11}$

Castration-resistant prostate cancer (CRPC) was defined by progression of disease (by PSA or radiographs) despite a castrate level of testosterone. PSA50 was defined as PSA decline of $\geq 50 \%$ from baseline PSA level prior to pembrolizumab initiation. PSA progression was defined as $25 \%$ increase from nadir PSA (confirmed by a second rising PSA at least 3 weeks later) per Prostate Cancer Working Group 3. Overall response rate was defined by RECIST 1.1. Patients were censored at the time of last follow-up. The cut-off date for analysis was April 202020.

\section{RESULTS}

During the study period, clinical G360 testing was performed for 460 patients with advanced prostate cancer by participating center collaborators; 405 of these patients had at least one ctDNA alteration identified. Of these, MSI-high status was identified in 15 patients (3.7\%). Additional clinical information was not available for one patient. The final analysis included a total of 14 metastatic prostate cancer patients, 12 (86\%) of whom were Caucasian with median age 69 (55-88). The median follow-up
Table 1 Baseline characteristics of metastatic CRPC patients treated with pembrolizumab

\begin{tabular}{ll}
\hline Characteristic & $\mathbf{n}=9$ patients \\
\hline Age, median (range), years & $68(57-88)$ \\
\hline Race, $\mathrm{n}(\%)$ & \\
\hline African-American & $1(11)$ \\
\hline Asian & $1(11)$ \\
\hline White & $7(78)$ \\
\hline Gleason grade group, $\mathrm{n}(\%)$ & \\
\hline 2 & $1(11)$ \\
\hline 4 & $2(22)$ \\
\hline 5 & $6(67)$ \\
\hline Location of metastases, $\mathrm{n}(\%)$ & \\
\hline Bones & $5(56)$ \\
\hline Soft-tissue & $1(11)$ \\
\hline Liver & $1(11)$ \\
\hline Lymph nodes & $3(33)$ \\
\hline Initial PSA, ng/dL, median (range) & $29.3(3.4-266)$ \\
\hline Prior lines of therapy for mCRPC, $\mathrm{n}(\%)$ & \\
\hline 0 & $1(11)$ \\
\hline 1 & $3(33)$ \\
\hline 2 & $3(33)$ \\
\hline 3 & $1(11)$ \\
\hline 4 & $1(11)$ \\
\hline Type of prior oncological therapies, $\mathrm{n}(\%)$ & \\
\hline Abiraterone & $6(67)$ \\
\hline Enzalutamide & $3(33)$ \\
\hline Ketoconazole & $1(11)$ \\
\hline Apalutamide & $1(11)$ \\
\hline Docetaxel & $2(22)$ \\
\hline Cabazitaxel & $1(11)$ \\
\hline Lutetium-177-PSMA-617 & $1(11)$ \\
\hline
\end{tabular}

CRPC, castration-resistant prostate cancer; mCRPC, metastatic CRPC.

was 2.6 years since metastatic disease was diagnosed and 12/14 patients developed CRPC. From the total MSI-H cohort $(\mathrm{n}=14)$, five patients did not receive immune checkpoint inhibitors due to death $(n=2)$, metastatic castration-naïve disease $(n=2)$ or insurance denial $(n=1)$. A total of nine patients with metastatic CRPC (mCRPC) with $56 \%$ bone, $33 \%$ nodal, $11 \%$ liver and $11 \%$ soft-tissue metastases and a median PSA of $29.3 \mathrm{ng} / \mathrm{dL}$ were treated with pembrolizumab after a median of $16.0(0.7-20.6)$ months after diagnosis of mCRPC and a median of 2 (0-4) prior lines of therapy for CRPC (table 1).

Of the nine patients that received pembrolizumab, drug was administered for an estimated median of $9.9(95 \%$ CI 1.0 to 18.8$)$ months. Four patients (44\%) achieved PSA50 after a median of 4 (3-12) weeks after treatment 


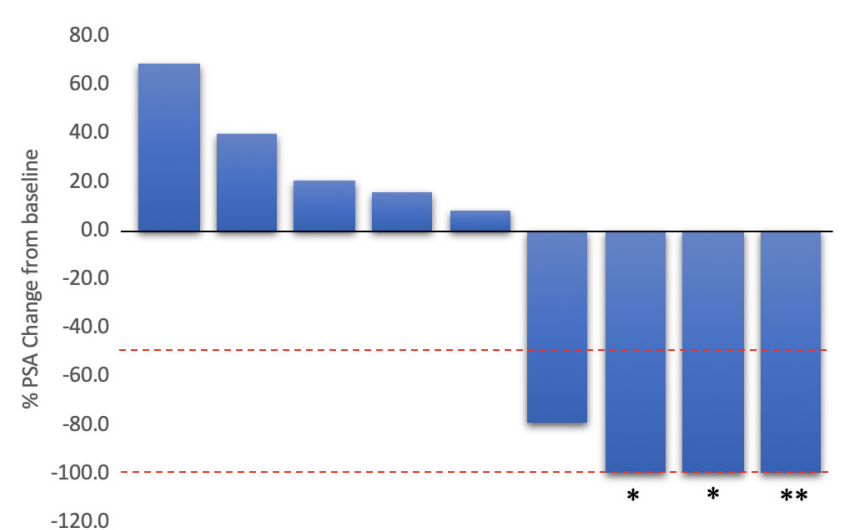

Figure 1 Best PSA change from baseline in mCRPC patients treated with pembrolizumab $(\mathrm{N}=9)$. " partial response, ${ }^{* *}$ complete response

initiation including three patients with $>99 \%$ PSA decline (figure 1).

Among the patients evaluable for radiographic response $(n=5)$, the overall response rate was $60 \%$ with one complete response (CR) and two partial responses (PR). One patient had a CR in lymph nodes while two patients had PR in lymph nodes. All responding patients had a PSA50. Two patients progressed on therapy. Best response was observed after a median of 3.3 (1.4-7.6) months and the responses were observed in lymph nodes $(\mathrm{n}=3)$ and liver $(\mathrm{n}=1)$.

At time of cut-off, all patients who had received pembrolizumab were alive; four patients were still on pembrolizumab, while four patients discontinued therapy due to progressive disease and one due to COVID-19 infection. All four progressors had evidence of both PSA and radiographic progression. Enzalutamide, docetaxel and radium 223 were the systemic therapies of choice for patients who progressed on pembrolizumab.

Clinically significant adverse events considered related to pembrolizumab by the treating physician were reported in one patient that required systemic corticosteroids (total dose daily of $20 \mathrm{mg}$ prednisone) for grade 2 arthralgias and grade 1 rash, and no treatment interruption or hospitalization was required.

All patients treated with pembrolizumab had cfDNA NGS testing done while having mCRPC. Testing was initially done a median of 13.7 (0.9-23.3) months after developing mCRPC and $0.6(0.3-6.8)$ months prior to starting pembrolizumab.

Considering the full MSI-H cohort $(\mathrm{n}=14)$, characterized genomic alterations were also found in the following (selected) genes: TP53 (64\%), AR (57\%), ARID1A (36\%), PTEN (36\%), BRCA1 (29\%), BRCA2 (21\%), PIK3CA (21\%), FGFR1/2 (21\%), ATM (14\%) and CDK12 (7\%). The median maximum mutant allele fraction on G360 in the cohort was $15.6 \%$ (range $3.34 \%-74 \%$ ). The median number of (SNVs; inclusive of both non-synonymous and synonymous alterations) identified by G360 in this cohort was 14.5 (5-48) and the median number of deletion mutation (indels) identified was $3.5(0-8)$.
Half of the patients with PSA50 had both MSI-H and pathogenic alterations in BRCA1 and BRCA2 detected by their G360 assays. One patient with PR had BRCA1, $B R C A 2$ and ATM mutations. There were no CDK12 alterations among responders.

MSI-H was detected in all three patients with available tumor tissue NGS. No germline genomic alterations were found in the two patients who underwent separate germline testing.

\section{DISCUSSION}

To our knowledge, this is the first case series reporting the clinical activity of pembrolizumab for MSI-H mCRPC identified by a cfDNA assay. This dataset consists of patients with predominately bone and nodal metastases and previously exposed to novel hormonal therapies.

While the efficacy of PD-1 inhibitors for unselected mCRPC is modest, ${ }^{3}$ durable and profound responses (PSA and radiographic) were observed in nearly half of the MSI-H tumors, consistent with prior reports in prostate and other tumor types. ${ }^{1518}$ Despite the inclusion of MSI-H/dMMR testing and pembrolizumab treatment for mCRPC with MSI-H/dMMR in the second line and beyond in the national guidelines, ${ }^{19}$ one patient could not be treated with pembrolizumab due to insurance limitations.

Although in small numbers, DNA repair defects in combination with MSI-H were associated with the responses to pembrolizumab, which supports their potential role as predictive biomarkers. ${ }^{20}$ Whether there is a synergy between anti-PD-1/PD-L1 agents and poly ADP ribose polymerase (PARP) inhibitors is being further explored. ${ }^{21} 22$

This case series might reflect a generalized practice of ordering a liquid biopsy after progression to MCRPC and after exposure to novel hormonal therapies, where the benefit of the remaining therapies is more limited. In most cases, the use of pembrolizumab was favored prior to the use of chemotherapy, which is frequently considered in routine practice.

Limited tumor tissue, insufficient quality/quantity and inability to assess current genomic landscape using archival tumor samples are known limitations in prostate cancer genomic assessment. Importantly, there is clear evidence of acquired MSI-H phenotype developing as prostate cancer advances and liquid biopsies can be of significant importance to overcome all of these limitations. ${ }^{18}$ Not all MMR mutations are truncal, and in some cases the root cause of MSI-H status remains unclear. This dataset provides evidence that the use of cfDNA NGS assays in clinical practice is feasible, has direct clinical implications and yields therapeutic response which is supported by the short period of time observed between testing and initiation of pembrolizumab therapy and subsequent responses. It is reassuring that the cfDNA assay used in this study has been validated with very high concordance, sensitivity and specificity and with a limit of detection of $0.1 \%$ tumor content for MSI-H status as well as additional genomic alterations with potential therapeutic implications. ${ }^{11} 17$ This dataset is concordant with other tumor datasets supporting 
the cfDNA testing as an appropriate surrogate marker for MSI status in men with mCRPC.

The prevalence of cfDNA MSI-H was 3.7\%, slightly higher than the previously reported prevalence of $2.3 \%(55 / 2358)$ of prostate cancer samples from the large pan-cancer validation study of this assay's MSI-H detection in plasma $\mathrm{cfDNA}^{11}$ and similarly consistent with reported prevalence of $3.1 \%$ and $3.8 \%$ in two other studies of MSI status in metastatic prostate cancer. ${ }^{123}$ By contrast, a lower prevalence of MSI-H $(0.6 \%)$ was noted in primary prostate carcinomas, based on The Cancer Genome Atlas (TCGA) dataset that included 497 patients. $^{24}$

The relatively short follow-up and small size of this cohort is due to the limited period of time in which plasma MSI-H testing has been clinically available. However, the clinical implications of confirmed treatment outcomes even in heavily pretreated patients, based on this cfDNA biomarker, warrant quick report of this early cohort.

Other PD-1/PD-L1 inhibitors are being tested in combination with different therapies to expand the use of these agents beyond MSI-H/dMMR. Examples include the combination of nivolumab with ipilimumab (CheckMate 650) or atezolizumab plus cabozantinib (COSMIC-021) where promising efficacy signals were observed in specific subgroups of patients. ${ }^{25}{ }^{26}$ In addition, other immune interventions such as genetic vaccination (NCT04041310) or adaptive cell therapies (NCT03935893) are currently under investigation for patients with MSI-H tumors.

At this time, several questions related to the optimal setting for PD-1 inhibitors in the advanced disease state, the role of intermittent therapy for those who achieve durable and profound responses, the emergence of additional biomarkers (such as tumor mutational burden or TMB, as well as individual positive and negative predictive genomic biomarkers) and the potential synergic activity when combined with other effective anticancer therapies, are being explored.

The limitations of this study include the retrospective nature of this analysis, the small number of patients treated with pembrolizumab and the selection bias associated with limited access to novel therapies under current development. The G360 data does not report TMB, thus, the association of detected MSI-H status with this potentially important biomarker is not assessed. However, in MSI-H assay validation studies, the median number of SNVs and indels detected in MSI-H samples were significantly higher than in microsatellite stable samples. ${ }^{11}$ The median number of those alteration types in the cohort presented here were even higher than that seen in the MSI-H plasma validation cohort (14.5 SNVs and 3.5 indels), suggesting that these cases might also be consistent with increased mutational burden.

\section{CONCLUSIONS}

In conclusion, the use of a well-validated cfDNA NGS liquid biopsy to identify metastatic prostate cancer patients with MSI-H is feasible in clinical practice and may overcome the obstacles associated with prostate cancer tumor tissue for timely genomic assessment. The robust activity of pembrolizumab in selected patients supports the generalized testing for MSI-H.

\section{Author affiliations}

${ }^{1}$ Deming Department of Medicine, Tulane University, New Orleans, Louisiana, USA

${ }^{2}$ Department of Medical Oncology, University of Utah, Salt Lake City, Utah, USA

${ }^{3}$ Department of Medical Oncology and Experimental Therapeutics, City of Hope Comprehensive Cancer Center, Duarte, California, USA

${ }^{4}$ Department of Medicine, University of Arizona Arizona Cancer Center, Tucson, Arizona, USA

${ }^{5}$ Department of Oncology, Mayo Clinic, Scottsdale, Arizona, USA

${ }^{6}$ Division of Hematology, Oncology, and Blood and Marrow Transplant, University of lowa, lowa City, lowa, USA

${ }^{7}$ Genitourinary Oncology Program, Division of Hematology, Oncology and Blood and Marrow Transplantation, The University of lowa, lowa City, lowa, USA

${ }^{8}$ Guardant Health Inc, Redwood City, California, USA

${ }^{9}$ Department of Oncology, Massachusetts General Hospital, Boston, Massachusetts, USA

Twitter Pedro Barata @PBarataMD and Alan Bryce @AlanBryce9

Contributors Concept: $\mathrm{PB}$ and $\mathrm{OS}$. Data collection: PB, RN, BG, HB, AB, RG, CS and PS. Data analysis and interpretation: all authors.

Funding The authors have not declared a specific grant for this research from any funding agency in the public, commercial or not-for-profit sectors.

Competing interests PB has a consulting/advisory role (Institution) with Exelixis, Caris, Bayer, Janssen, EMD/Serono, Pfizer, Astellas, Dendreon, Clovis and Sanofi. Contracted Research (Institution) from Seattle Genetics, BlueEarth Diagnostics, Nektar, AstraZeneca and Seattle Genetics. NA has a consulting/advisory role with Astellas, Astra Zeneca, Bayer, Bristol Myers Squibb, Clovis, Eisai, Eli Lilly, EMD Serono, Exelixis, Foundation Medicine, Genentech, Janssen, Merck, Nektar, Novartis, Pfizer, Pharmacyclics and Seattle Genetics. Contracted Research (Instituion) from Astra Zeneca, Bavarian Nordic, Bayer, BN immunotherapeutics, Bristol Myers Squibb, Calithera, Celldex, Clovis, Eisai, Eli Lilly, EMD Serono, Exelixis, Genentech, Glaxo Smith Kline, Immunomedics, Janssen, Medivation, Merck, Nektar, New Link Genetics, Novartis, Pfizer, Prometheus, Rexahn, Roche, Sanofi, Seattle Genetics, Takeda and Tracon. HB has a consulting/advisory role with Endocyte, Celgene, Guardant360, Tracon. Honoraria: SirTex, Bayer. Speaker Bureau: Guardant360. LK is employee of Guardant Health. OS has a consulting/advisory role with AAA Astellas, Astrazeneca, Bayer, Blue Earth Diagnostics, EMD Serono, Endocyte, Pfizer, Progenics, Sanofi, Invitae, Merck, Novartis, Janseen, Constellation, Dendreon, BMS, Bravarin Nordic, Clovis, Myriad, Noria Therapeutics, Noxopharm, Point Biopharma and Tenebio. Contracted Research from Innocrin, Sotio. He also serves as consultant on NCI Scientific Board Counselors and is a cochairman of GU Committee at NRG.

\section{Patient consent for publication Not required.}

Ethics approval This retrospective study was approved by an institutional review board with a waiver of consent for the analysis of deidentified data.

Provenance and peer review Not commissioned; externally peer reviewed.

Data availability statement All data relevant to the study are included in the article or uploaded as online supplementary information. All data generated or analyzed during this study are included in this published article.

Open access This is an open access article distributed in accordance with the Creative Commons Attribution Non Commercial (CC BY-NC 4.0) license, which permits others to distribute, remix, adapt, build upon this work non-commercially, and license their derivative works on different terms, provided the original work is properly cited, appropriate credit is given, any changes made indicated, and the use is non-commercial. See http://creativecommons.org/licenses/by-nc/4.0/.

\section{ORCID iDs}

Pedro Barata http://orcid.org/0000-0002-8890-2951

Neeraj Agarwal http://orcid.org/0000-0003-1076-0428

Rohan Garje http://orcid.org/0000-0002-7244-5602

\section{REFERENCES}

1 Darvin P, Toor SM, Sasidharan Nair V, et al. Immune checkpoint inhibitors: recent progress and potential biomarkers. Exp Mol Med 2018;50:1-11. 
2 Hargadon KM, Johnson CE, Williams CJ. Immune checkpoint blockade therapy for cancer: an overview of FDA-approved immune checkpoint inhibitors. Int Immunopharmacol 2018;62:29-39.

3 Antonarakis ES, Piulats JM, Gross-Goupil M, et al. Pembrolizumab for treatment-refractory metastatic castration-resistant prostate cancer: Multicohort, open-label phase II KEYNOTE-199 study. J Clin Oncol 2020;38:395-405

4 Cook N, Hansen AR, Siu LL, et al. Early phase clinical trials to identify optimal dosing and safety. Mol Oncol 2015;9:997-1007.

5 Li K, Luo H, Huang L, et al. Microsatellite instability: a review of what the oncologist should know. Cancer Cell Int 2020;20:16

6 Shia J, Stadler Z, Weiser MR, et al. Immunohistochemical staining for DNA mismatch repair proteins in intestinal tract carcinoma: how reliable are biopsy samples? Am J Surg Pathol 2011;35:447-54.

7 Murphy KM, Zhang S, Geiger T, et al. Comparison of the microsatellite instability analysis system and the Bethesda panel for the determination of microsatellite instability in colorectal cancers. $J$ Mol Diagn 2006;8:305-11.

8 Niu B, Ye K, Zhang Q, et al. MSIsensor: microsatellite instability detection using paired tumor-normal sequence data. Bioinformatics 2014;30:1015-6.

9 Salipante SJ, Scroggins SM, Hampel HL, et al. Microsatellite instability detection by next generation sequencing. Clin Chem 2014;60:1192-9.

10 Georgiadis A, Durham JN, Keefer LA, et al. Noninvasive detection of microsatellite instability and high tumor mutation burden in cancer patients treated with PD-1 blockade. Clin Cancer Res 2019;25:7024-34.

11 Willis J, Lefterova MI, Artyomenko A, et al. Validation of microsatellite instability detection using a comprehensive plasma-based genotyping panel. Clin Cancer Res 2019;25:7035-45.

12 Abida W, Cheng ML, Armenia J, et al. Analysis of the prevalence of microsatellite instability in prostate cancer and response to immune checkpoint blockade. JAMA Oncol 2019:5:471-478.

13 Robinson D, Van Allen EM, Wu Y-M, et al. Integrative clinical genomics of advanced prostate cancer. Cell 2015;162:454.

14 Reichert ZR, Urrutia J, Alumkal JJ. Microsatellite instability as an emerging biomarker for checkpoint inhibitor response in advanced prostate cancer. JAMA Oncol 2019;5:478-9.

15 Le DT, Uram JN, Wang $\mathrm{H}$, et al. Pd-1 blockade in tumors with mismatch-repair deficiency. N Engl J Med 2015;372:2509-20.
16 Marcus L, Lemery SJ, Keegan P, et al. Fda approval summary: pembrolizumab for the treatment of microsatellite instability-high solid tumors. Clin Cancer Res 2019;25:3753-8.

17 Odegaard JI, Vincent JJ, Mortimer S, et al. Validation of a plasmabased comprehensive cancer genotyping assay utilizing orthogonal tissue- and plasma-based methodologies. Clin Cancer Res 2018;24:3539-49.

18 Abida W, Cheng ML, Armenia J, et al. Analysis of the prevalence of microsatellite instability in prostate cancer and response to immune checkpoint blockade. JAMA Oncol 2019;5:471-8.

19 National comprehensive cancer network: prostate cancer. version 1, 2020. Available: https://www.nccn.org/professionals/physician_gls/ pdf/prostate.pdf

20 Mouw KW, Goldberg MS, Konstantinopoulos PA, et al. Dna damage and repair biomarkers of immunotherapy response. Cancer Discov 2017;7:675-93.

21 Vinayak S, Tolaney SM, Schwartzberg L, et al. Open-Label clinical trial of Niraparib combined with pembrolizumab for treatment of advanced or metastatic triple-negative breast cancer. JAMA Oncology 2019;5:1132-40.

22 Friedlander M, Meniawy T, Markman B, et al. Pamiparib in combination with tislelizumab in patients with advanced solid tumours: results from the dose-escalation stage of a multicentre, open-label, phase 1a/b trial. Lancet Oncol 2019;20:1306-15.

23 Mayrhofer M, De Laere B, Whitington T, et al. Cell-Free DNA profiling of metastatic prostate cancer reveals microsatellite instability, structural rearrangements and clonal hematopoiesis. Genome Med 2018;10:85.

24 Cortes-Ciriano I, Lee S, Park W-Y, et al. A molecular portrait of microsatellite instability across multiple cancers. Nat Commun 2017;8:15180.

25 Sharma P, Pachynski RK, Narayan V, et al. Initial results from a phase II study of nivolumab (NIVO) plus ipilimumab (IPI) for the treatment of metastatic castration-resistant prostate cancer (mCRPC; CheckMate 650. . American Society of Clinical Oncology, 2019: 37. 142

26 Agarwal N, Loriot Y, McGregor BA. Cabozantinib (C) in combination with atezolizumab (a) in patients (PTS) with metastatic castrationresistant prostate cancer ( $M C R P C)$ : results of cohort 6 of the COSMIC-021 study. American Society of Clinical Oncology, 2020. 